\title{
The effects of competence and social class on degree of modeling of self-reward patterns $^{1}$
}

HERBERT A. COLLE AND HELEN L. BEE UNIVERSITY OF WASHINGTON

Competence of model, competence of subject, and social class were varied in an experiment on the acquisition of standards of excellence. The major finding was a social class effect; lower class $S$ s, in comparison to middle class $S s$, showed little adoption of a model's standard of excellence. It was suggested that lower class Ss do not adopt high standards set by a model.

A number of experimenters have explored the use of modeling techniques in the development of consistent self-reward patterns in children (Bandura \& Kupers, 1964; Bandura \& Whalen, 1966; Mischel \& Liebert, 1966; Bee \& Colle, 1967). In the typical procedure the child observes a model playing a bowling game and sees the model reward himself verbally and with candy only for scores above some specified standard. When the child is then given the opportunity to play the game with free access to the candy, he tends to match the standards set by the model. Since "standard setting" is a crucial feature of "achievement behavior," the paradigm seems relevant for the exploration of the origins of standards of excellence in children. The present study was an attempt to examine the effects of two major variables on the standard setting behavior of children in this experimental setting: (a) the degree of match or mismatch between the perceived competence of the model and $S$ at the game, and (b) the social class (SES) of the child. Earlier work (Bee \& Colle, 1967) suggested that both of these variables should have an impact on the standard setting observed in the child. It was predicted that a high degree of match between the perceived competence of the child and the model would be associated with high standard setting, while mismatch would be associated with low standard setting. In addition, it was expected that lower social class Ss would show less consistent standard setting than higher social class Ss. Method

The Ss were 80 boys, aged 8-13 (mean age 10.5) enrolled in a public school in Seattle, Washington. All Ss were tested, during school hours, by the same male $\mathrm{E}$.

The apparatus consisted of a miniature bowling alley, $9 \mathrm{ft}$ in length, raised $2 \mathrm{ft}$ off the floor. The pin area was screened from the $S$ so that knowledge of actual proficiency could be prevented. A counter above the pin area showed a two digit integer on each trial; the series of scores for both $\mathrm{E}$ and $\mathrm{S}$ were programmed prior to the start of the experimental session.
A factorial design consisting of four levels of social class (based on fathers' occupations), two levels of model competence, and two levels of $S$ competence was used.

Information about fathers' occupations was obtained from all children in grades 3-6 in the school, with all scorable descriptions categorized on a 7 point scale (Warner, Meeker, \& Eells, 1949) with 1 representing professional occupations and 7, unskilled work. The four levels of social class were: Group 1, scale points 1 to 4 (mean 2.4, median 3.0); Group 2, scale point 5; Group 3, scale point 6; and Group 4, scale points 6 and 7 (mean 6.7, median 7.0). These groups, which involved a greater differentiation in the lower part of the scale, reflected the greater number of lower class Ss available in the school population. Within each of these groups, Ss were randomly assigned to one of the four experimental conditions.

Ss were tested individually in a two-part experimental session. Part 1 consisted of 18 training trials, played by $E$, with each trial consisting of one throw. For scores above 20, E made a positive statement (e.g., "That's a good shot!"') and took candy while saying "I'll take a piece of candy." For scores below 20, E made a negative statement and did not take candy. All Ss observed the model receive nine scores above the standard of 20 and nine scores below. Model competence was varied with the scores below the standard. High model competence Ss observed an E receiving two scores below 10 and seven scores between 11 and 20. Low model competence Ss observed an E receiving seven scores below 10 and two scores between 11 and 20 .

Following the training trials, after being reminded that he could take a piece of candy any time he wanted to, $\mathrm{S}$ was asked to play the game and was given 18 test trials. All Ss received nine scores above the standard in the test trials, but high and low competence was again varied in scores below the standard for $S$. The high and low competence series of scores were the same for training and testing, except that the order was re-randomized for the test trials.

Results

Each S's pattern of self-reward was transformed into a standard setting score by taking the percentage of self-reward for scores above 20 and subtracting from this the percentage of self-reward for scores below 20. Thus, each $\mathrm{S}$ was assigned a single score which represented the extent to which his self-reward pat- 
tern followed that demonstrated by the model. An analysis of variance, with model competence, subject competence, and social class as the between-subject varibles was then computed.

There was a significant main effect of $S$ competence $(F=4.3, p<.05)$, with Ss receiving low competence scores showing higher standard setting than Ss receiving high competence scores. The main effect of model competence did not approach significance, nor did the interaction between $S$ and model competence. A main effect of social class $(F=4.6, p<.01$ ) was obtained, with higher social class Ss showing greater standard setting. The mean standard setting scores for the four SES groups were $31.1,12.0,2.4$, and 8.4 for Groups 1 through 4, respectively. The median standard setting scores were $33,0,0$, and 0 for the same four groups. Social class did not interact significantly with either model or S competence.

Since the standard setting scores were not normally distributed, the main effects were further checked with Kruskal-Wallis rank tests. Using this analysis, the variable of $S$ competence yielded an $H$, corrected for ties, of $3.0(p<.10, d f=1)$ while the social class variable yielded a corrected $H$ of $14.9(p<.01, d f=3)$. When the three lower class groups together were compared with the highest SES group, a corrected $\mathrm{H}$ of $11.8(\mathrm{p}<.001, \mathrm{df}=1)$ was obtained.

\section{Discussion}

The results of the present experiment provided only partial support for the initial expectations. The anticipated relationship between model and $S$ competence was not obtained, but there was a significant social class effect.

In the previous study (Bee \& Colle, 1967) in which both high and low standards had been used with both modeling and direct reward procedures, the highstandard modeling group had shown little standard setting. Since, in that group, there was a discrepancy between the competence of the model and that of the child, the variable of competence was suggested as an explanatory concept. The results of the present study do not support that possibility. However, a second suggestion, namely that lower class Ss (who predominated in the previous study as well) may not adopt high standards set by the model, receives some support from the present experiment. All Ss in the present study observed a model setting a high standard, and the level of standard setting for the lower class Ss in the present data is comparable to the level observed in the high-standard modeling group in the previous study. If one can assume that middle and upper class children typically adopt high standards for their own behavior, then the standard set by the model in the present experiment presumably more closely approximated a type of average standard for these Ss. For the lower class Ss, on the other hand, the high standard set by the model may have been more rigorous a standard than they typically adopted for themselves.

That the social class effect is not being produced by IQ differences is demonstrated by the nonsignificant $(r=+.17)$ correlation, based on $70 \mathrm{Ss}$, between LorgeThorndike Verbal IQ and level of standard setting. Excluding IQ as a factor, and given the fact that in the previous experiment lower class Ss had responded with moderate standard setting to a low standard, it seems reasonable to presume that it is the level of standard, in interaction with social class, which is producing the effect.

If this interpretation is correct, there are clear implications for the use of modeling as an instructional technique for lower class children. In addition, this finding may suggest caution in generalizing the results of previous experiments on modeling, all of which have apparently used middle or upper class children as Ss.

The S competence effect, which was constant across SES groups, appears best explained in terms of a contrast effect. The above-standard scores were markedly higher than the below-standard scores for the low competence Ss, and may have stood out more clearly as good scores.

References

BANDURA, A., \& KUPERS, C. J. Transmission of patterns of selfreinforcement through modeling. J. abnorm. soc. Psychol., 1964, $69,1-9$.

BANDURA, A., \& WHALEN, C. J. KUPERS. The influence of antecedent reinforcement and divergent modeling cues on patterns of selfreward. J. pers. soc. Psychol., 1966, 3, 373-382.

BEE, H. L., \& COLLE, H. A. The origins of standards of excellence: I. Modeling vs direct reinforcement. Paper read at meetings of Soc. Res. Child Develpm., New York, March, 1967.

MISCHEL, W., \& LIEBERT, R. M. Effects of discrepancies between observed and imposed reward criteria on their acquisition and transmission. J. pers. soc, Psychol., 1966, 3, 45-53.

WARNER, W. L., MEEKER, M., \& EELLS, K. Social Class in America Chicago: Science Research Associates, 1949.

Note

1. This research was supported by the Graduate School Research Fund, University of Washington. 